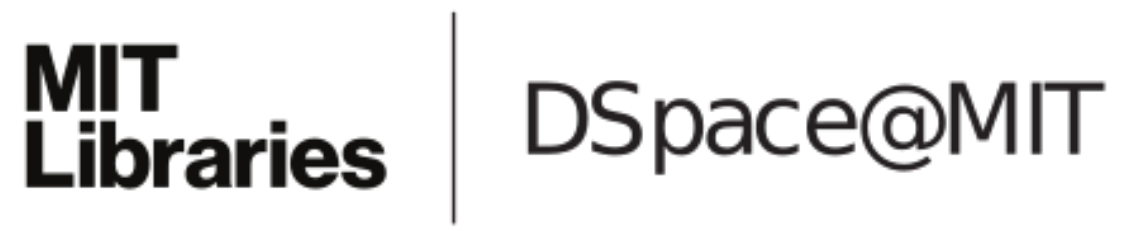

\author{
MIT Open Access Articles
}

High-velocity micro-particle impact on gelatin and synthetic hydrogel

The MIT Faculty has made this article openly available. Please share how this access benefits you. Your story matters.

Citation: Veysset, David et al. "High-velocity micro-particle impact on gelatin and synthetic hydrogel." Journal of the Mechanical Behavior of Biomedical Materials 86 (October 2018): 71-76 (C) 2018 Elsevier

As Published: http://dx.doi.org/10.1016/j.jmbbm.2018.06.016

Publisher: Elsevier BV

Persistent URL: https://hdl.handle.net/1721.1/122055

Version: Author's final manuscript: final author's manuscript post peer review, without publisher's formatting or copy editing

Terms of use: Creative Commons Attribution-NonCommercial-NoDerivs License 


\section{Author's Accepted Manuscript}

High-velocity micro-particle impact on gelatin and synthetic hydrogel

David Veysset, Steven E. Kooi, A.A. Maznev, Shengchang Tang, Aleksandar S. Mijailovic, Yun Jung Yang, Kyle Geiser, Krystyn J. Van Vliet, Bradley D. Olsen, Keith. A. Nelson

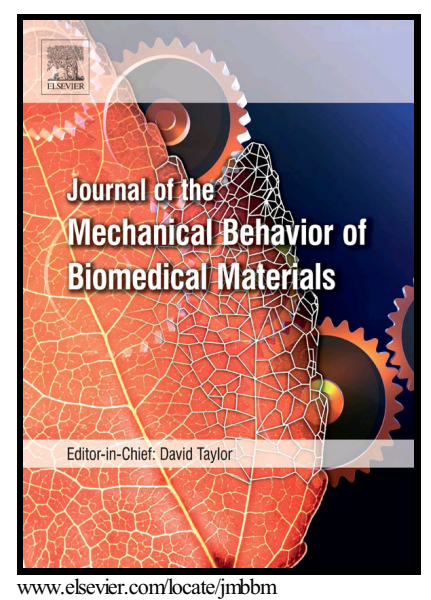

PII: $\quad$ S1751-6161(18)30473-9

DOI: $\quad$ https://doi.org/10.1016/j.jmbbm.2018.06.016

Reference: JMBBM2837

To appear in: Journal of the Mechanical Behavior of Biomedical Materials

Received date: 9 April 2018

Revised date: 29 May 2018

Accepted date: 9 June 2018

Cite this article as: David Veysset, Steven E. Kooi, A.A. Maznev, Shengchang Tang, Aleksandar S. Mijailovic, Yun Jung Yang, Kyle Geiser, Krystyn J. Van Vliet, Bradley D. Olsen and Keith. A. Nelson, High-velocity micro-particle impact on gelatin and synthetic hydrogel, Journal of the Mechanical Behavior of Biomedical Materials, https://doi.org/10.1016/j.jmbbm.2018.06.016

This is a PDF file of an unedited manuscript that has been accepted for publication. As a service to our customers we are providing this early version of the manuscript. The manuscript will undergo copyediting, typesetting, and review of the resulting galley proof before it is published in its final citable form. Please note that during the production process errors may be discovered which could affect the content, and all legal disclaimers that apply to the journal pertain. 


\title{
High-velocity micro-particle impact on gelatin and synthetic hydrogel
}

David Veysset, ${ }^{\mathrm{a}, \mathrm{b}, *}$, Steven E. Kooi ${ }^{\mathrm{a}}$, A. A. Maznev ${ }^{\mathrm{a}, \mathrm{b}}$, Shengchang Tang ${ }^{\mathrm{c}}$, Aleksandar S. Mijailovic $^{\mathrm{d}}$, Yun Jung Yang ${ }^{\mathrm{c}}$, Kyle Geiser ${ }^{\mathrm{e}}$, Krystyn J. Van Vliet ${ }^{\mathrm{e}}$, Bradley D. Olsen ${ }^{\mathrm{a}, \mathrm{c}}$, and Keith. A. Nelson ${ }^{\mathrm{a}, \mathrm{b}}$

${ }^{a}$ Institute for Soldier Nanotechnologies, Massachusetts Institute of Technology, Cambridge, Massachusetts 02139, USA

bepartment of Chemistry, Massachusetts Institute of Technology, Cambridge, Massachusetts 02139, USA

${ }^{\mathrm{c}}$ Department of Chemical Engineering, Massachusetts Institute of Technology, Cambridge, Massachusetts 02139, USA

${ }^{\mathrm{d}}$ Department of Mechanical Engineering, Massachusetts Institute of Technology, Cambridge, Massachusetts 02139, USA

'Department of Materials Science and Engineering, Massachusetts Institute of Technology, Cambridge, Massachusetts 02139, USA

*Correspondence to author. dveysset@mit.edu (D. Veysset)

\begin{abstract}
The high-velocity impact response of gelatin and synthetic hydrogel samples is investigated using a laser-based microballistic platform for launching and imaging supersonic micro-particles. The micro-particles are monitored during impact and penetration into the gels using a high-speed multi-frame camera that can record up to 16 images with nanosecond time resolution. The trajectories are compared with a Poncelet model for particle penetration, demonstrating good agreement between experiments and the model for impact in gelatin. The model is further validated on a synthetic hydrogel and the applicability of the results is discussed. We find the strength resistance parameter in the Poncelet model to be two orders of magnitude higher than in macroscopic experiments at comparable impact velocities. The results open prospects for testing high-rate behavior of soft materials on the microscale and for guiding the design of drug delivery methods using accelerated microparticles.
\end{abstract}


Keywords:

high-velocity impact; high-speed imaging; gelatin; hydrogel; penetration.

\section{Introduction}

The high-speed micro-impact response of soft tissues has direct implications in two fields, which at first sight may seem contradictory. On one hand, studying the high-rate mechanical behavior of tissues or tissue simulants can help in the understanding of ballistic and explosive-related injuries and lead to better forensic interpretation in cases that involve such injuries. For instance, small fragments originating from explosions can cause severe tissue damage injuries, characterized as secondary blast injuries. Among the ejected objects, micro-debris, which typically travels at supersonic velocities, can cause serious injury as it can penetrate deep into tissue and is hard to detect using standard medical imaging methods (Centeno et al., 2014; Hill et al., 2001; Kane et al., 2009; Wolf and Bucknell, 2010). On the other hand, in biolistics, microparticles launched at supersonic velocities are used as carriers to deliver therapeutic substances into cells and tissues (Klein et al., 1987; Sanford et al., 1987). In both fields, most studies have been based on post-mortem or ex-vivo analysis wherein the final penetration depth of the micro-particle is often the only quantity that is measured (Menezes et al., 2005; Mitchell, 2003) and the full impact history such as the dynamic extent of tissue damage and tissue recovery remains unknown. A capability to study impact dynamics in real time would be greatly beneficial for elucidating the mechanics of soft tissues under high velocity micro-particle impact. Such capability has now become available as a result of the development of the laser-induced particle impact test (LIPIT) platform, in which an intense short laser pulse is used to accelerate microparticles to supersonic velocities (Lee et al., 2012, 2014; Thevamaran et al., 2016; Xie et al., 2017), and individual particle impact events are observed with a high-frame-rate camera (Hassani-Gangaraj et al., 2018, 2017, Veysset et al., 2017, 2016).

In this work, we use the LIPIT platform to study the impact of microparticles on gelatin and synthetic hydrogel samples at velocities up to $\sim 1500 \mathrm{~m} / \mathrm{s}$. Gelatin has been widely used in ballistic studies as a mechanical simulant for soft biological tissues, including skin, muscle, brain, and other soft internal organs, depending on the gelation solid concentration (Ferry, 1948; Guha et al., 2010; Koene and Papy, 2011; Thali et al., 2002; Yoon et al., 2015). In addition to being cheap and easily manufactured, gelatin is transparent, which enables the visualization of the micro-particle penetrations and the high-rate deformation of the gelatin samples. We also study microparticle impact on a protein-based synthetic hydrogel that is of potential interest for injectable tissue engineering therapies (Olsen et al., 2010) and responsive hydrogels (Glassman et al., 2013). Micro-particle penetration trajectories are compared with a semi-empirical Poncelet model, which is found to describe the experimental data quite well.

\section{Materials and methods}




\subsection{Sample preparation}

Gelatin samples with three different concentrations $(2.5,5$, and $10 \mathrm{wt} \%)$ were prepared following the standard method proposed by Jussila (Jussila, 2004) using gelatin powder from bovine and porcine bones (Honeywell, Inc.). After preparation, the gelatin samples were allowed to solidify for 24 hours at room temperature and then for 24 hours at $+4^{\circ} \mathrm{C}$, for final solidification and conditioning. The preparation of $\mathrm{PC}_{10} \mathrm{P}$ protein hydrogels was performed according to published procedures (Olsen et al., 2010). Briefly, the recombinant proteins were expressed in E.coli., purified by metal-affinity Ni-NTA chromatography, dialyzed against water and lyophilized. Protein powders were then dissolved in $100 \mathrm{mM}$ phosphate buffer $(\mathrm{pH}$ 7.6) at a gel concentration of $10 \%(\mathrm{w} / \mathrm{v})$ at $4^{\circ} \mathrm{C}$ overnight. The gel was mixed several times with a micro-spatula to ensure homogenous mixing, pressed into the mold, and left at $4^{\circ} \mathrm{C}$ for at least $4 \mathrm{~h}$ to allow self-healing. All batches were stored in a fridge at $4^{\circ} \mathrm{C}$ before tests and were taken out of the fridge to be immediately tested in a room at ambient temperature. Quasi-static rheological properties of the gelatin samples are available in the supplementary information. Rheological data for the $\mathrm{PC}_{10} \mathrm{P}$ samples are available in (Olsen et al., 2010). All tested specimens were 10-mm tall, 10-mm wide, and 2-mm thick.

\subsection{Microparticle impact test}

The laser-induced particle impact test (LIPIT) apparatus was initially designed by Lee et al. (Lee et al., 2012) and recently expanded to allow for high-speed imaging of impact dynamics by Veysset et al. (Veysset et al., 2016). An intense laser pulse (10-ns duration, 532-nm wavelength) is focused onto a launching assembly that consists of a glass substrate $(210-\mu \mathrm{m}$ thick), a gold film (60-nm thick), a polyurea film (30- $\mu \mathrm{m}$ thick), and silica spheres $(7.38-\mu \mathrm{m}$ diameter) deposited on top of the polyurea film. Upon laser ablation of the sacrificial gold layer, the polyurea film quickly expands and ejects the silica microparticles to high velocities into free space (see Fig. 1). By adjusting the laser energy from 0.30 to $20.0 \mathrm{~mJ}$, silica particles were accelerated from $\sim 200$ to $\sim 1500 \mathrm{~m} / \mathrm{s}$. 


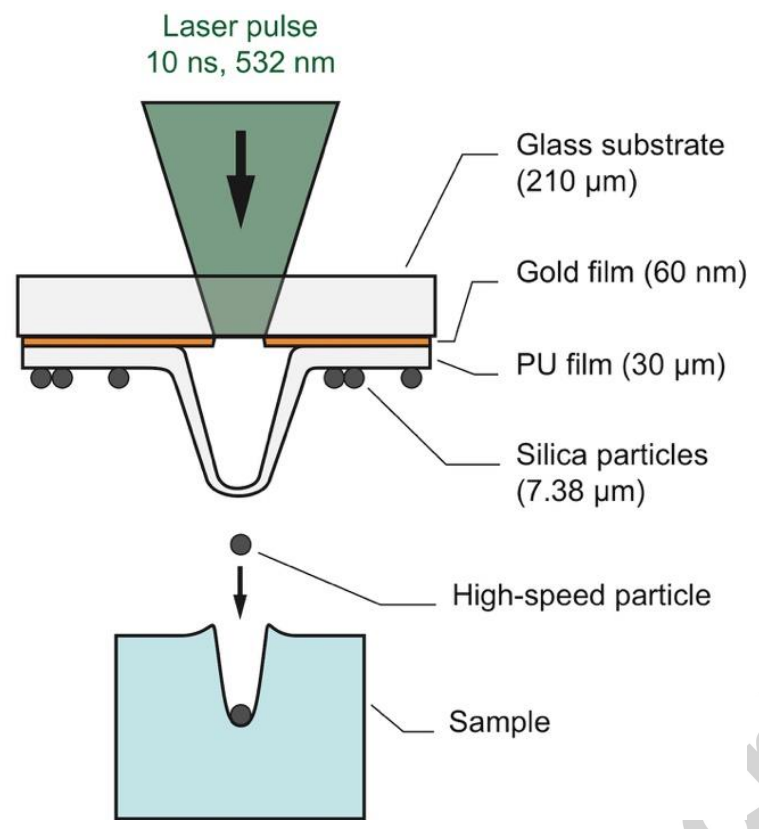

Fig. 1 - Schematic of the experiment. Upon laser ablation of a gold film, a polyurea film quickly expands and accelerates microparticles to supersonic velocities up to $\sim 1500 \mathrm{~m} / \mathrm{s}$. The subsequent particle impact and penetration in gelatin are imaged in transmission using a $\mu$ s laser pulse and a high-speed camera. The distance between the launching assembly and the sample is approximately $750 \mu \mathrm{m}$. (1-column figure, color)

The impact events were recorded using a 640-nm wavelength, 15- $\mu$ s-duration illumination laser (Cavilux, Specialised Imaging) and a high-frame-rate camera (SIMX16, Specialised Imaging). The camera can record 16-frame videos with adjustable exposure durations and inter-frame times that can be as short as $3 \mathrm{~ns}$. The acquisition trigger was set so that a few frames captured the incident particle before the impact in order to measure the impact velocity. The subsequent frames captured the particle penetration in the gel as illustrated in Fig. 2, which shows a typical image sequence of an impact on a $10 \mathrm{wt} \%$ gelatin sample at a velocity of $1290 \mathrm{~m} / \mathrm{s}( \pm 15 \mathrm{~m} / \mathrm{s})$ (see supplementary video S1 for full-field view).

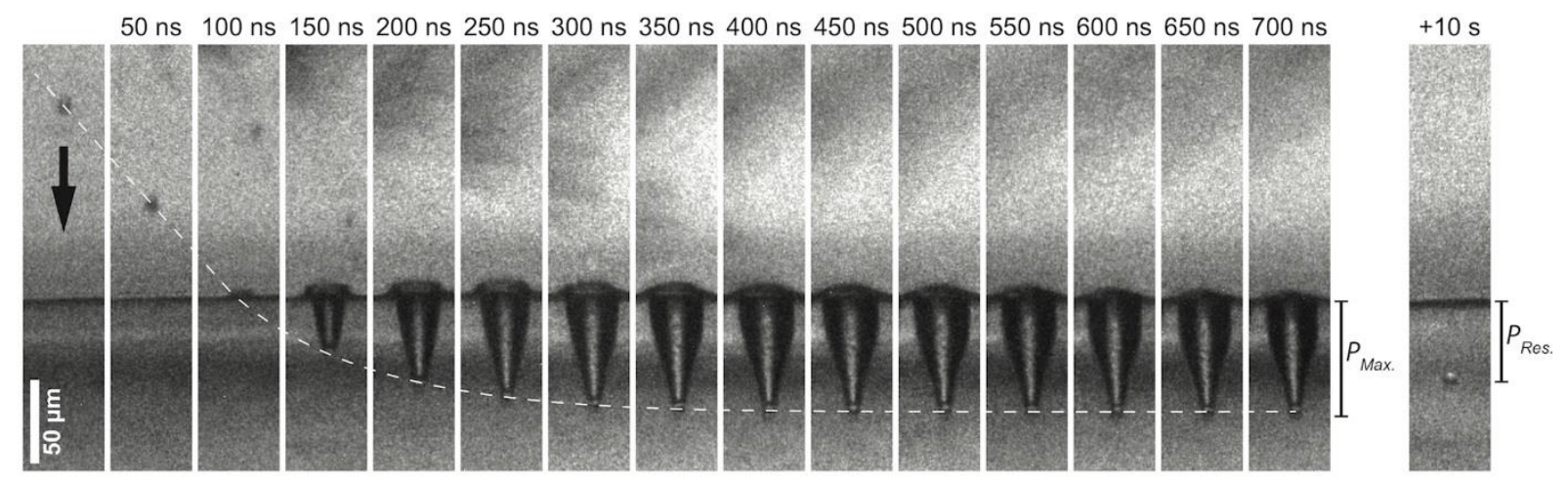


Fig. 2 - Multi-frame sequence showing a high-velocity impact on $10 \mathrm{wt} \%$ gelatin at $1290 \mathrm{~m} / \mathrm{s}$. The particle hits the sample surface $100 \mathrm{~ns}$ after the first frame is acquired. A small splash forms and develops upwards. The maximum penetration is reached within 200-250 ns after impact. The white dashed line illustrates the position of the particle versus time. An image taken a few seconds after the impact events shows the residual penetration of the particle. The interframe time is shown at the top of the frames and the exposure time is $10 \mathrm{~ns}$ for all frames. (2-column figure, black and white)

As can be observed on the third frame of Fig. 2, for an impact at $1290 \mathrm{~m} / \mathrm{s}$, a splash forms as the particle hits the surface. Subsequently, the particle penetrates in the sample opening a large air cavity in its wake. Such cavity opening has been frequently observed for high-velocity impacts on viscoelastic materials (Akers and Belmonte, 2006; Liu et al., 2012; Mrozek et al., 2015) and more traditionally in water entry problems (Truscott et al., 2014). After slowing down, the particle reaches its maximum penetration, $P_{\text {Max }}$, approximately 200 ns (corresponding to 4 frames) after impact. Finally, the particle is pulled back upwards to reach its final position, or residual penetration $P_{\text {Res }}$, as evidenced by an image that was taken about $10 \mathrm{~s}$ after impact. The residual penetration, while not being the focus of this investigation as it occurs on much longer timescales, is of interest as the backward motion of deeply penetrating particles is not yet fully understood (Akers and Belmonte, 2006).

Particle trajectories as well as maximum and residual penetrations were extracted from image sequences such that shown as in Fig. 2 for velocities ranging from $\sim 200 \mathrm{~m} / \mathrm{s}$ to $\sim 1500 \mathrm{~m} / \mathrm{s}$. More details regarding the launching assembly preparation, the optical setup, and the image analysis can be found in (Veysset, 2016; Veysset et al., 2016).

\subsection{Poncelet model for particle penetration}

As a particle penetrates into a sample, it is subjected to a variety of forces including inertial, viscous, capillary, gravitational, elastic, and strength terms. To determine the relative contributions of these terms one can evaluate the dimensionless parameters that are the Reynolds number $R e$ (inertia vs. viscosity), the Weber number We (inertia vs. capillarity), the Froude number $F r$ (inertia vs. gravity), and the elastic Froude number $F e$ (inertia vs. elasticity) (Akers and Belmonte, 2006; Katsuragi, 2016). These parameters also define the studied impact regime. These quantities are defined as $R e=\rho_{s} v L / \mu, W e=\rho_{s} v^{2} L / \sigma, F r=v^{2} / g L$, and $F e=$ $\Delta \rho v^{2} / G$, where $\rho_{s}$ is the density of the fluid (sample), $v$ is the velocity of the object relative to the medium, $L$ is the characteristic dimension of the object, $\mu$ is the dynamic viscosity of the fluid, $\sigma$ is the fluid surface tension, $g$ is the standard gravity, $\Delta \rho$ is the difference in density between the particle and the fluid, and $G$ is the instantaneous shear storage modulus of the fluid. 
Here, under our conditions with $\rho_{s} \sim 1000 \mathrm{~kg} / \mathrm{m}^{3}, v \sim 1000 \mathrm{~m} / \mathrm{s}, L \sim 10 \mu \mathrm{m}, \mu \sim 10^{-2}$ Pa.s for gelatin (Liu et al., 2012), $\sigma \sim 10^{-2} \mathrm{~N} / \mathrm{m}$ (Johnston and Peard, 1925), $g \sim 10 \mathrm{~m} / \mathrm{s}^{2}, \Delta \rho \sim 850 \mathrm{~kg} / \mathrm{m}^{3}$, and $G \sim 10^{6}$ $\mathrm{Pa}$ (Swain et al., 2014), we have $\mathrm{Re} \sim 10^{3}, \mathrm{We} \sim 10^{6}, \mathrm{Fr} \sim 10^{10}$, and $\mathrm{Fe} \sim 10^{3}$. In the present high Weber, high Froude, and high elastic Froude numbers regime, the force, $F$, that is experienced by the particle can be expressed as a polynomial of the velocity $v$ :

$F=B_{2} v^{2}+B_{0}$

where $B_{2}$ and $B_{0}$ represent the inertial drag and the strength resistance term, respectively. These equation is known as the Poncelet equation (Allen et al., 1957). The inertial drag can be expressed as

$B_{2} v^{2}=\frac{1}{2} C_{D} \rho_{S} A v^{2}$

where $C_{D}$ is the drag coefficient, $\rho_{s}$ is the density of the sample, $A$ is cross-sectional area of the particle and $v$ is the particle velocity. $C_{D}$ depends on the particle shape and the Reynolds number (Schlichting and Gersten, 2017). Under the present flow conditions $\left(R e \sim 10^{3}\right)$, the coefficient of drag for a sphere is equal to 0.4 (Schlichting and Gersten, 2017). We consider this value for $C_{D}$ to be constant under our experimental conditions. The validity of this assumption is discussed later in the text.

The resistance term $B_{0}$ can be expressed as

$B_{0}=A R$

where $R$ is the strength resistance (Akers and Belmonte, 2006; Dehn, 1987). An explicit timedependent solution to the Poncelet equation is given by Segletes (Segletes, 2008):

$z(t)=\frac{m}{B_{2}}\left[\ln \cos \left(\frac{\sqrt{B_{2} B_{0}}}{m}\left(t_{f}-t\right)\right)-\ln \cos \left(\frac{\sqrt{B_{2} B_{0}}}{m} t_{f}\right)\right]$

where $z(t)$ is the particle coordinate in the target as a function of time and $t_{f}$ is the final time at which the particle is stopped upon reaching the maximum penetration distance.

$t_{f}=\frac{m}{\sqrt{B_{2} B_{0}}} \tan ^{-1}\left(v_{0} \sqrt{\frac{B_{2}}{B_{0}}}\right)$

where $m$ is the mass of the particle and where $v_{0}$ is the impact velocity. We define the maximum penetration depth, $P_{\operatorname{Max}}$, to be equal to the maximum coordinate $z\left(t_{f}\right)$ to which we add half a particle diameter in order to account for the particle 'nose' contribution. Hence, 
$P_{\text {Max }}=z\left(t_{f}\right)+\frac{d}{2}=\frac{2}{3} \frac{\rho_{p} d}{\rho_{s} C_{D}} \ln \left(\frac{\rho_{s} C_{D} v_{0}^{2}}{2 R}+1\right)+\frac{d}{2}$

Material properties used in the Poncelet model are listed in Table 1.

Table 1 - Summary of particle and sample properties.

\begin{tabular}{llll}
\hline Silica sphere particle & Gelatin sample densities & PC $_{10}$ P sample density \\
\hline$d=7.38 \mu \mathrm{m}( \pm 0.08 \mu \mathrm{m})$ & Gelatin 2.5 $\mathrm{wt} \%:$ & $\rho_{s}=1000 \mathrm{~kg} / \mathrm{m}^{3}$ & $\rho_{s}=1020 \mathrm{~kg} / \mathrm{m}^{3}$ \\
$\rho_{p}=1850 \mathrm{~kg} / \mathrm{m}^{3}$ & Gelatin 5 wt $\%$ & $\rho_{s}=1010 \mathrm{~kg} / \mathrm{m}^{3}$ & \\
& Gelatin 10 $\mathrm{wt} \%:$ & $\rho_{s}=1030 \mathrm{~kg} / \mathrm{m}^{3}$ & \\
\hline
\end{tabular}

From (Winter, 1975), based on gelatin concentrations.

\section{Results and discussion}

\subsection{Impact on gelatin}

The particle trajectory was extracted from the image sequence in Fig. 2 and is shown in Fig. 3(a). A series of impacts was performed with varying particle velocity from $\sim 200 \mathrm{~m} / \mathrm{s}$ to $\sim 1500 \mathrm{~m} / \mathrm{s}$ and the normalized maximum and residual penetrations, $P_{\text {Max }} / d$ and $P_{\text {Res }} / d$ respectively, are reported in Fig. 3(b). For each gelatin specimen, multiple impacts were performed on the specimen as the sample dimensions were large compared to the particle size and the volume of sample affected by impact. Between each laser shot, the target sample was moved laterally by at least $400 \mu \mathrm{m}$ to an intact area. When the edge of the sample was reached, another sample from the gelatin batch was cut and placed as new target. To limit gelatin dehydration, specimens were replaced every 10 minutes. In the event where multiple particles were accelerated at the same time (from the same laser shot) toward the target, we only selected and analyzed impacts that would be at least $40 \mu \mathrm{m}$ away from adjacent impacts. No significant difference in penetration results was observed for gelatin specimen coming from different batches following the same sample preparation procedure. Despite data scattering from shot to shot, there is a clear trend, i.e., an impact at higher velocity resulted in deeper penetration, as expected. The maximum penetration data was fitted using the Poncelet solution for the maximum penetration Eq. (6) with the resistance $R$ as the only fitting parameter, which was in turn used to calculate the complete particle trajectory using Eq. (4) for the particular impact shown in Fig. 2. This trajectory is shown in Fig. 3(a) (solid line) and agrees well with the experimental data. Thus, the deceleration and the time to maximum penetration $t_{f}$ can be accurately predicted using the Poncelet model. In addition, because the inertial term is predominant at high velocity, the good agreement between the experiment and the model in the early stage of penetration validates our choice for $C_{D}=0.4$ (see Eq. (2)). Further discussion on the choice for $C_{D}$ can be found in the supplementary information. 
(a)

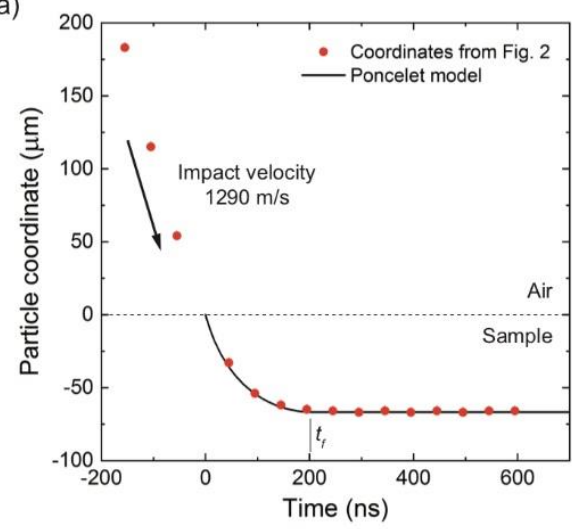

(b)

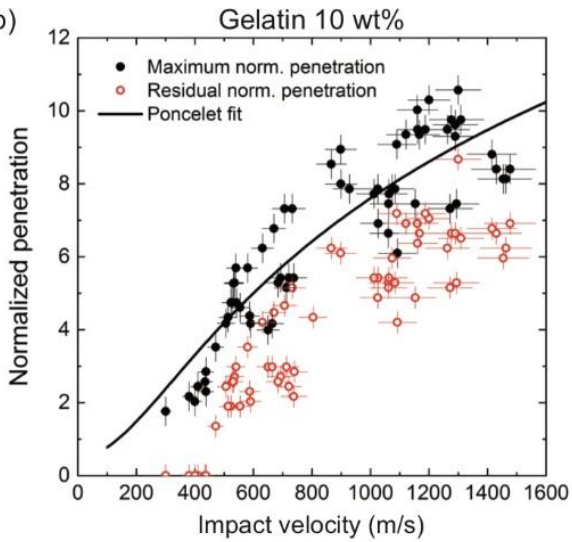

Fig. 3 - (a) Particle trajectory corresponding to the impact shown in Fig. 2, where the particle impacts a $10 \mathrm{wt} \%$ gelatin sample at $1290 \mathrm{~m} / \mathrm{s}$. The trajectory is compared with the Poncelet model using Eq. (4) and the fitted parameter $R$ obtained from the maximum penetration data. (b) Normalized maximum and residual penetrations as a function of impact velocity for impacts on $10 \mathrm{wt} \%$ gelatin. The maximum penetration data are fitted with the Poncelet model and the resistance $R$ as a parameter. (1.5-column figure, color)

Similar impact tests were conducted on $5 \mathrm{wt} \%$ and $2.5 \mathrm{wt} \%$ gelatin samples. Likewise, the resistance parameter was extracted for both concentrations after fitting the experimental results with the Poncelet model (see Fig. 4). Even though we cannot draw a direct correlation between the resistance and the yield strength, one would expect that a higher strength would lead to a higher resistance and here, indeed, the resistance increases as the water content decreases (Fig. 4c). We also note, that $R=21 \mathrm{MPa}$ for $10 \mathrm{wt} \%$ gelatin is comparable to high strain rate (2000$3000 \mathrm{~s}^{-1}$ ) strength measurements of $10 \mathrm{wt} \%$ gelatin by Kwan and Subhash, which were on the order of 2-6 MPa and increased with strain rate (Kwon and Subhash, 2010). In a comparable study, using a shock tube-based system for microparticle acceleration, Kendall analyzed penetration data in human skin in view of the Poncelet model and found a similar resistance value of the order of $10^{7} \mathrm{~Pa}$ (Kendall, 2002).
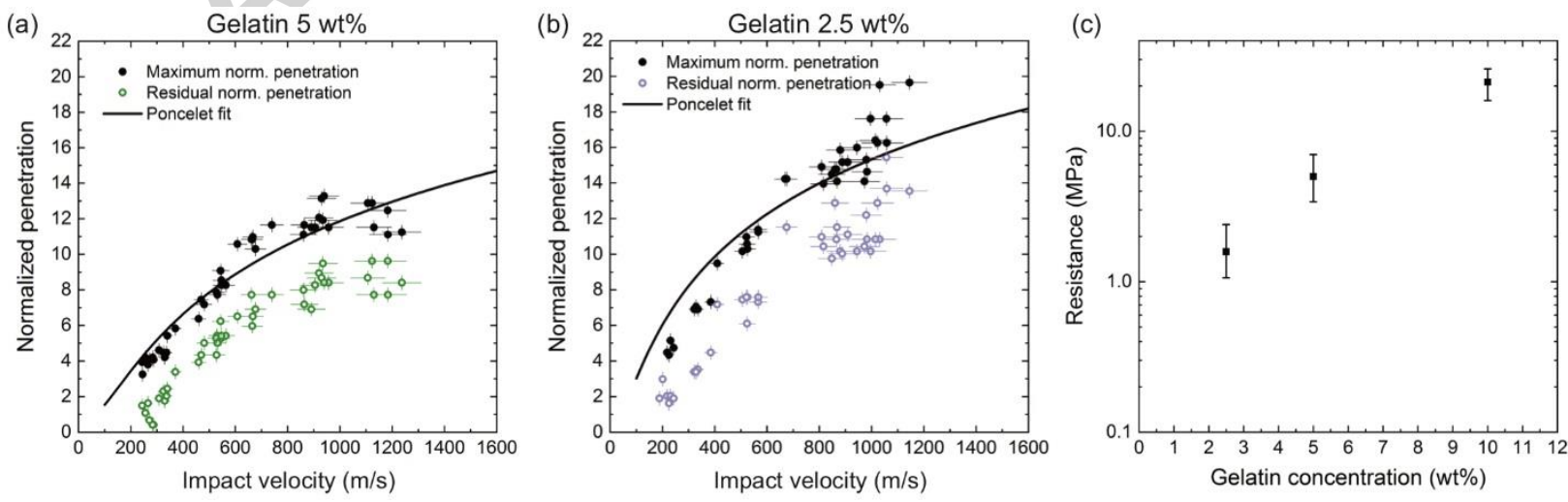
Fig. 4 - $(a, b)$ Normalized maximum and residual penetrations as a function on impact velocity for impacts on (a) $5 \mathrm{wt} \%$ gelatin and (b) $2.5 \mathrm{wt} \%$ gelatin (b). (c) Resistance values, obtained after penetration data fitting, as a function of gelatin concentration. The error bars represent the range for the $R$ values enclosing the experimental data. The coefficient of determination for the 10 $\mathrm{wt} \%, 5 \mathrm{wt} \%$, and $2.5 \mathrm{wt} \%$ gelatin concentrations are $0.80,0.90$, and 0.87 , respectively. (2column figure, color)

The reason for the scatter in the penetration data (shown for instance in Fig. 3(b)) is unclear. The uncertainties in particle speed and maximum penetration depth for individual impacts are small compared to the data scatter. On possibility, we hypothesize, could be dehydration of the waterbased gels resulting in local variation in the physical properties of the gels. In the present study, though, samples were replaced every 10 minutes in order to limit sample drying and our data does not suggest any correlation between the time of the experiment and the penetration depth.

\subsection{Impact on synthetic hydrogel}

The results presented above establish that the Poncelet model can describe the particle trajectory in gelatin, and perhaps predict the impact response of other soft materials in the same impact regime (high Reynolds, Weber, Froude, and elastic Froude numbers), where the penetration is governed by inertial and resistance effects. This predictive capability becomes particularly advantageous when dealing with novel materials that are often available in small quantities and/or are expensive to produce, which is not the case for gelatin. To verify this, we conducted impact experiments on a special type of engineered protein hydrogel, termed as $\mathrm{PC}_{10} \mathrm{P}(\mathrm{Olsen}$ et al., 2010), in order to demonstrate that, from few experiments, one could estimate the unknown parameter $R$ and then predict the impact behavior of the same material under difference impact conditions, i.e, at a different speed and/or with a different particle.

For the $\mathrm{PC}_{10} \mathrm{P}$ sample, we assumed no a priori knowledge of the hydrogel mechanical properties apart from its density $\left(\sim 1020 \mathrm{~kg} / \mathrm{m}^{3}\right)$. Fig. 5(a) shows an image sequence of an impact at $530 \mathrm{~m} / \mathrm{s}$ $( \pm 15 \mathrm{~m} / \mathrm{s})$ using a silica sphere particle. The impact closely resembles what was observed with gelatin: a splash forms and the particle penetrates deeply in the sample opening a large air cavity behind. Fig. 5(b) shows an impact of a copper particle (12- $\mu \mathrm{m}$ diameter, purchased from Alfa Aesar) on the same sample at a similar velocity of $435 \mathrm{~m} / \mathrm{s}( \pm 15 \mathrm{~m} / \mathrm{s})$. Because the copper particle is about 5 times denser and 1.6 times larger than the silica particle, it penetrated much deeper into the sample and ultimately exited the imaging field of view (last frame of Fig. 5(b)) with a maximum penetration depth exceeding $300 \mu \mathrm{m}$. We also note a larger splash following impact and the emergence of thin jets at cusp about 400-600 ns after impact. Only one experiment using the copper projectile was successful. 


\section{(a)}

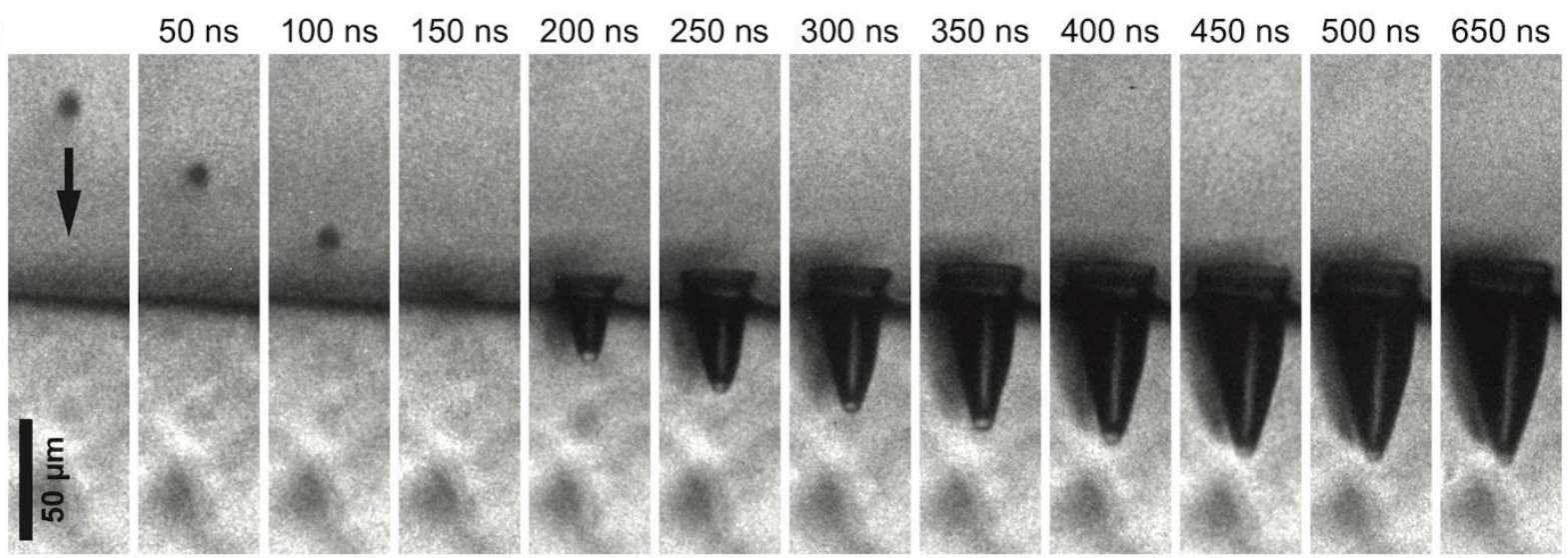

(b) $200 \mathrm{~ns} 400 \mathrm{~ns}$
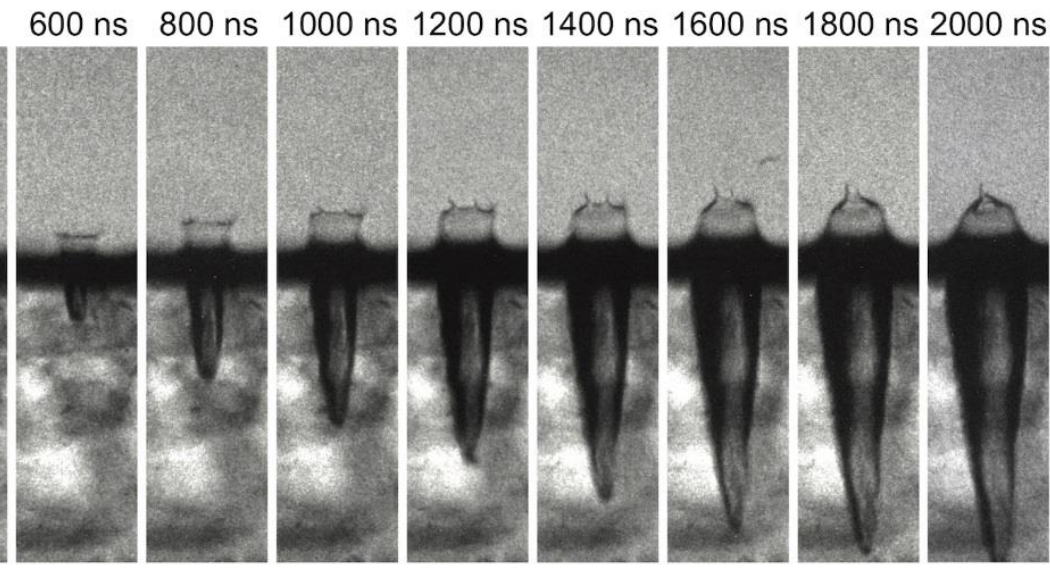

Fig. 5 - Multi-frame sequences showing high-velocity impacts on a $\mathrm{PC}_{10} \mathrm{P}$ sample. (a) Impact of a silica sphere particle $(7.38-\mu \mathrm{m}$ diameter) at $530 \mathrm{~m} / \mathrm{s}$. (b) Impact of a copper particle $(12-\mu \mathrm{m}$ diameter) at $435 \mathrm{~m} / \mathrm{s}$. The exposure time is $10 \mathrm{~ns}$ for all frames. (2-column figure, black and white)

Fig. 5(a) shows the penetration data obtained from a limited number of experiments on the $\mathrm{PC}_{10} \mathrm{P}$ specimen, from which a value of $R=2.4 \mathrm{MPa}$ was fitted. Both particle trajectories were extracted from the image sequences and are shown in Fig. 6(b,c). The fitted $R$ parameter was then used to calculate the predicted trajectories of the silica and copper particle in the sample. The good agreement between the experimentally-measured trajectories and the predicted ones can be seen in Figs. 6(b,c). We additionally plotted particle trajectories corresponding to $\pm 50 \%$ variations in $R$, which corresponds to the uncertainty on $R$ obtained for the gelatin samples. Although we could not capture the ultimate stages of penetration for the copper particle Figs. 6 (c), the effects of resistance became visible after $\sim 1000 \mathrm{~ns}$ and the parameter $R$ provides a good match with the observed deceleration. To observe the full penetration a field of view of about 1 $\mathrm{mm}$ would have been necessary. However, this would have reduced the resolution of the images making it difficult to locate the particle accurately. 

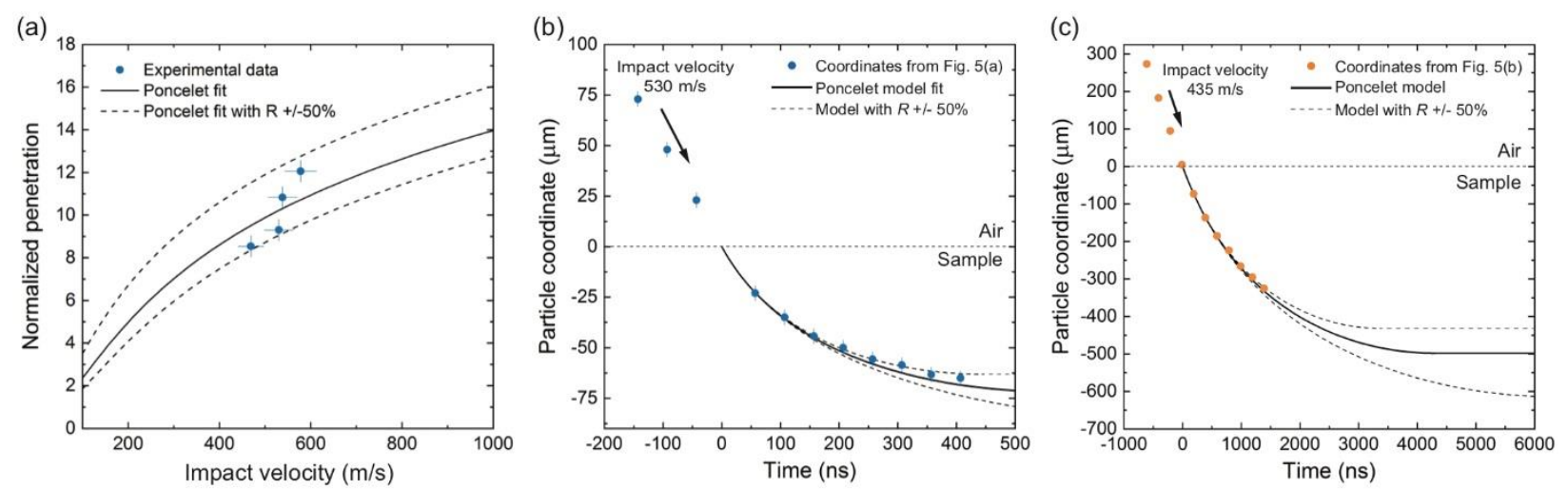

Fig. 6 - (a) Normalized maximum and residual penetrations as a function of impact velocity for impacts on $\mathrm{PC}_{10} \mathrm{P}$. The maximum penetration data are fitted with the Poncelet model and the resistance $R$ as a parameter. (b) Particle trajectory corresponding to the impact on $\mathrm{PC}_{10} \mathrm{P}$ shown in Fig. 5(a) using a silica particle. (c) Particle trajectory corresponding to the impact on $\mathrm{PC}_{10} \mathrm{P}$ shown in Fig. 5(b) using a copper particle. The trajectories are predicted using the Poncelet model (Eq. (4)) and using the $R$ parameter obtained from the penetration data shown in (a) along with trajectories corresponding to $R \pm 50 \%$. (2-column figure, color)

These results suggest that the Poncelet model can reasonably predict particle impact at supersonic velocities not only at the macroscale, as previously demonstrated (Guzman et al., 2014; Segletes, 2008), but also at the microscale. This can prove particularly useful when studying novel materials for which mechanical properties at high strain rates are mostly unknown.

It should finally be noted that in our study, both the drag coefficient $C_{D}$ and the resistance $R$ were, as a first approximation, considered constant, and the model with fixed values of $C_{D}$ and $R$ agrees well within the velocity range of this study. In reality, $C_{D}$, which depends on the Reynolds number, changes with velocity (Schlichting and Gersten, 2017). The velocity dependence on $C_{D}$ should therefore be included in the model to describe impacts over a wider range of Reynolds number (Segletes, 2008). Likewise, the resistance $R$, which is related to the strength of the material, depends on the strain rate (Segletes, 2008). We nevertheless note that our data do not show a variation in $R$ over the range of studied velocities where the strain rate varies by a factor of 5. However, Liu et al. estimated the resistance of a $10 \mathrm{wt} \%$ gelatin sample for a mm-sized steel particle supersonic impact (also high Reynold, Weber, Froude, and elastic Froude numbers) to be of the order of $10^{5} \mathrm{~Pa}$ for a characteristic strain rate of $10^{6} \mathrm{~s}^{-1}$ whereas in the present case $R$ is of the order of $10^{7} \mathrm{~Pa}$ for a characteristic strain rate of $10^{8} \mathrm{~s}^{-1}$. Consequently, the values of $R$ that we found in this study are only valid for a typical strain rate of $10^{8} \mathrm{~s}^{-1}$. Reciprocally, values for $R$ obtained in macroscopic experiments cannot be used to predict microscopic impacts. 


\section{Conclusions}

We have studied real-time microparticle impacts on gel samples using a laser-induced particle impact test platform. In addition to observing the entire particle trajectory, the present experimental method enables the direct in-situ visualization of material response at high strain rate, including cavity dynamics, at previously unexplored spatiotemporal scales. The experimental method also allows the production of a large quantity of data using a minimum quantity of the sample material, which is particularly beneficial for the development of novel materials and the validation of predictive models. From impact image sequences, micro-particle trajectories were extracted and compared with a simple Poncelet model using a single fitting parameter, the resistance, which was evaluated for three gelatin concentrations. The Poncelet model was further tested on a synthetic hydrogel sample using silica and copper particles launched at similar impact velocities. The results suggest that the model can reasonably predict micro-particle penetration and serve as the first step towards more elaborate models for deformation of soft materials at high strain rates. We also find that the resistance values, as described in the Poncelet model, obtained under high-speed microscopic impacts differ by orders of magnitude compared to high-speed macroscopic impacts, justifying the need for such in-situ experiments. We envision that an enhanced prediction of tissue or tissue-simulant responses under impact will guide the development of micrometer-sized drug delivery carriers and help better assess the threat of micro-debris in explosive-related injuries.

\section{Acknowledgments}

D.V. and S.E.K thank Michael J. Daniti, Pierre-Thomas Brun, and Bo Qing for fruitful discussions. A.A.M. appreciates stimulating discussions of biolistics with Dmitry Rinberg. This research was supported by the U.S. Army Research Office under contract W911NF-13-D-0001. Support for equipment was also provided through the Office of Naval Research DURIP Grant No. N00014-13-1-0676. The authors have no competing interests to declare.

\section{References}

Akers, B., Belmonte, A., 2006. Impact dynamics of a solid sphere falling into a viscoelastic micellar fluid. J. Nonnewton. Fluid Mech. 135, 97-108. doi:10.1016/j.jnnfm.2006.01.004

Allen, W.A., Mayfield, E.B., Morrison, H.L., 1957. Dynamics of a Projectile Penetrating Sand. J. Appl. Phys. 28, 370-376. doi:10.1063/1.1722750

Centeno, J., Rogers, D., van der Voet, G., Fornero, E., Zhang, L., Mullick, F., Chapman, G., Olabisi, A., Wagner, D., Stojadinovic, A., Potter, B., 2014. Embedded Fragments from U.S. Military Personnel - Chemical Analysis and Potential Health Implications. Int. J. Environ. Res. Public Health 11, 1261-1278. doi:10.3390/ijerph110201261

Dehn, J., 1987. A unified theory of penetration. Int. J. Impact Eng. 5, 239-248. doi:10.1016/0734-743X(87)90041-8 
Ferry, J.D., 1948. Mechanical Properties of Substances of High Molecular Weight. IV. Rigidities of Gelatin Gels; Dependence on Concentration, Temperature and Molecular Weight 1. J. Am. Chem. Soc. 70, 2244-2249. doi:10.1021/ja01186a074

Glassman, M.J., Chan, J., Olsen, B.D., 2013. Reinforcement of shear thinning protein hydrogels by responsive block copolymer self-assembly. Adv. Funct. Mater. 23, 1182-1193. doi:10.1002/adfm.201202034

Guha, R.A., Shear, N.H., Papini, M., 2010. Ballistic impact of single particles into gelatin: experiments and modeling with application to transdermal pharmaceutical delivery. J. Biomech. Eng. 132, 101003. doi:10.1115/1.4002428

Guzman, I.L., Iskander, M., Bless, S., Qi, C., 2014. Terminal depth of penetration of spherical projectiles in transparent granular media. Granul. Matter 16, 829-842. doi:10.1007/s10035014-0528-y

Hassani-Gangaraj, M., Veysset, D., Nelson, K.A., Schuh, C.A., 2018. In-situ observations of single micro-particle impact bonding. Scr. Mater. 145, 9-13. doi:10.1016/j.scriptamat.2017.09.042

Hassani-Gangaraj, M., Veysset, D., Nelson, K.A., Schuh, C.A., 2017. Melting Can Hinder Impact-Induced Adhesion. Phys. Rev. Lett. 119, 175701. doi:10.1103/PhysRevLett.119.175701

Hill, P.F., Edwards, D.P., Bowyer, G.W., 2001. Small Fragment Wounds: Biophysics, Pathophysiology and Principles of Management. J. R. Army Med. Corps 147, 41-51. doi:10.1136/jramc-147-01-04

Johnston, J.H., Peard, G.T., 1925. The Surface Tension of Gelatin Solutions. Biochem. J. 19, 281-9.

Jussila, J., 2004. Preparing ballistic gelatine - Review and proposal for a standard method. Forensic Sci. Int. 141, 91-98. doi:10.1016/j.forsciint.2003.11.036

Kane, M.A., Kasper, C.E., Kalinich, J.F., Kane, C.D.R.M.A., Usn, M.S.N., Kasper, C.E., Kalinich, J.F., 2009. Protocol for the assessment of potential health effects from embedded metal fragments. Mil. Med. 174, 265-269.

Katsuragi, H., 2016. Physics of Soft Impact and Cratering. doi:10.1007/978-4-431-55648-0

Kendall, M.A.F., 2002. The delivery of particulate vaccines and drugs to human skin with a practical, hand-held shock tube-based system. Shock Waves 12, 23-30. doi:10.1007/s001930200126

Klein, T.M., Wolf, E.D., Wu, R., Sanford, J.C., Klein, R.M., Wolf, E.D., Wu, R., Sanford, J.C., 1987. High-velocity microprojectiles for delivering nucleic acids into living cells. Nature 327, 70-73. doi:10.1038/327070a0

Koene, L., Papy, A., 2011. Towards a better, science-based, evaluation of kinetic non-lethal weapons. Int. J. Intell. Def. Support Syst. 4, 169. doi:10.1504/IJIDSS.2011.039548

Kwon, J., Subhash, G., 2010. Compressive strain rate sensitivity of ballistic gelatin. J. Biomech. 43, 420-425. doi:10.1016/j.jbiomech.2009.10.008

Lee, J.-H., Veysset, D., Singer, J.P., Retsch, M., Saini, G., Pezeril, T., Nelson, K.A., Thomas, E.L., 2012. High strain rate deformation of layered nanocomposites. Nat. Commun. 3, 1164. doi:10.1038/ncomms2166

Lee, J.J.-H., Loya, P.E., Lou, J., Thomas, E.L., 2014. Dynamic mechanical behavior of multilayer graphene via supersonic projectile penetration. Science 346, 1092-1096. doi:10.1126/science. 1258544

Liu, L., Fan, Y., Li, W., Liu, H., 2012. Cavity dynamics and drag force of high-speed penetration 
of rigid spheres into $10 \mathrm{wt} \%$ gelatin. Int. J. Impact Eng. 50, 68-75. doi:10.1016/j.ijimpeng.2012.06.004

Menezes, V., Takayama, K., Ohki, T., Gopalan, J., 2005. Laser-ablation-assisted microparticle acceleration for drug delivery. Appl. Phys. Lett. 87, 163504. doi:10.1063/1.2093930

Mitchell, T., 2003. A ballistic study of micro-particle penetration to the oral mucosa. Int. J. Impact Eng. 28, 581-599. doi:10.1016/S0734-743X(02)00150-1

Mrozek, R.A., Leighliter, B., Gold, C.S., Beringer, I.R., Yu, J.H., VanLandingham, M.R., Moy, P., Foster, M.H., Lenhart, J.L., 2015. The relationship between mechanical properties and ballistic penetration depth in a viscoelastic gel. J. Mech. Behav. Biomed. Mater. 44, 109120. doi:10.1016/j.jmbbm.2015.01.001

Olsen, B.D., Kornfield, J.A., Tirrell, D.A., 2010. Yielding behavior in injectable hydrogels from telechelic proteins. Macromolecules 43, 9094-9099. doi:10.1021/ma101434a

Sanford, J.C., Klein, T.M., Wolf, E.D., Allen, N., 1987. Delivery of substances into cells and tissues using a particle bombardment process. Part. Sci. Technol. 5, 27-37. doi:10.1080/02726358708904533

Schlichting, H., Gersten, K., 2017. Boundary-Layer Theory, 9th ed. Springer Berlin Heidelberg, Berlin, Heidelberg. doi:10.1007/978-3-662-52919-5

Segletes, S.B., 2008. Modeling the Penetration Behavior of Rigid Spheres Into Ballistic Gelatin.

Swain, M.V., Kieser, D.C., Shah, S., Kieser, J.A., 2014. Projectile penetration into ballistic gelatin. J. Mech. Behav. Biomed. Mater. 29, 385-392. doi:10.1016/j.jmbbm.2013.09.024

Thali, M.J., Kneubuehl, B.P., Zollinger, U., Dirnhofer, R., 2002. The "Skin-skull-brain model": A new instrument for the study of gunshot effects. Forensic Sci. Int. 125, 178-189. doi:10.1016/S0379-0738(01)00637-5

Thevamaran, R., Lawal, O., Yazdi, S., Jeon, S.-J., Lee, J.-H., Thomas, E.L., 2016. Dynamic creation and evolution of gradient nanostructure in single-crystal metallic microcubes. Science 354, 312-316. doi:10.1126/science.aag1768

Truscott, T.T., Epps, B.P., Belden, J., 2014. Water Entry of Projectiles. Annu. Rev. Fluid Mech. 46, 355-378. doi:10.1146/annurev-fluid-011212-140753

Veysset, D., 2016. Real-Time Observations of Materials under Dynamic Loading Conditions at the Micron Scale. MIT.

Veysset, D., Hsieh, A.J., Kooi, S., Maznev, A.A., Masser, K.A., Nelson, K.A., 2016. Dynamics of supersonic microparticle impact on elastomers revealed by real-time multi-frame imaging. Sci. Rep. 6, 25577. doi:10.1038/srep25577

Veysset, D., Hsieh, A.J., Kooi, S.E., Nelson, K.A., 2017. Molecular influence in high-strain-rate microparticle impact response of poly(urethane urea) elastomers. Polymer 123, 30-38. doi:10.1016/j.polymer.2017.06.071

Winter, J., 1975. The Material Properties of Gelatin Gels. Ballist. Res. Lab.

Wolf, J.M., Bucknell, A., 2010. Arthroscopic removal of improvised explosive device (IED) debris from the wrist: a case report. Mil. Med. 175, 68th Annual Meeting of the ASSH: Education through.

Xie, W., Alizadeh-Dehkharghani, A., Chen, Q., Champagne, V.K., Wang, X., Nardi, A.T., Kooi, S., Müftï, S., Lee, J.-H., 2017. Dynamics and extreme plasticity of metallic microparticles in supersonic collisions. Sci. Rep. 7, 5073. doi:10.1038/s41598-017-05104-7

Yoon, G.H., Mo, J.S., Kim, K.H., Yoon, C.H., Lim, N.H., 2015. Investigation of bullet penetration in ballistic gelatin via finite element simulation and experiment. J. Mech. Sci. Technol. 29, 3747-3759. doi:10.1007/s12206-015-0821-7 